Arq. Bras. Med. Vet. Zootec., v.69, n.4, p.908-914, 2017

\title{
Estenose esofágica em duas cadelas após ovário-histerectomia: relato de caso
}

\author{
[Esophageal stricture in two female dogs after ovariohysterectomy: case report] \\ M.G. Luciani, G. Biezus, H.M. Cardoso, T.R. Müller, P.E. Ferian, L.P. Souza, \\ D.S. Souza, R.A. Casagrande \\ Centro de Ciências Agroveterinárias - CAV-UDESC - Florianópolis, SC
}

\begin{abstract}
RESUMO
A estenose esofágica benigna é uma afecção rara em pequenos animais, comumente secundária a esofagites ulcerativas. O refluxo gastroesofágico, frequente durante procedimentos anestésicos, é a principal causa de esofagite grave, com consequente formação de cicatriz esofágica. O presente trabalho tem por objetivo descrever dois casos de estenose esofágica ocorrentes após ovário-histerectomia, com destaque para os procedimentos diagnósticos realizados. Em ambos os casos, a combinação dos sinais clínicos sugestivos e os achados de esofagograma e esofagoscopia foram determinantes. No primeiro caso, devido ao tempo avançado de desenvolvimento dos sinais clínicos, o paciente veio a óbito antes mesmo que a intervenção direta da região de estenose fosse realizada. Já no segundo, o procedimento de gastrostomia para melhor manejo alimentar, associado à dilatação esofágica via esofagoscopia e à terapia com medicamentos antiácidos, resultou em melhora clínica.
\end{abstract}

Palavras-chave: canino, esofagite, cicatriz esofágica, estenose esofágica, refluxo gastroesofágico

\begin{abstract}
Benign esophageal stricture is a rare affection in small animals, usually secondary to ulcerative esophagitis. Gastroesophageal reflux, frequent during anesthetic procedures, is the main cause of severe esophagitis with consequent formation of esophageal cicatrix. The objective of this work is to describe two cases of esophageal stricture occurring after ovariohysterectomy, highlighting the diagnostic procedures performed. In both cases, the combination of the suggestive clinical signs and findings from an esophagram and an esophagoscopy were determinants. In the first case, due to the advanced stage of the clinical signs, the patient died before direct interventions on the stenosis region were performed. But in the second case, the gastrostomy procedures for better feed management associated with esophageal dilatation by esophagoscopy and therapy with antacids resulted in clinical improvement.
\end{abstract}

Keywords: dog, esophagitis, esophageal cicatrix, esophageal stricture, gastroesophageal reflux

\section{INTRODUÇÃO}

A estenose esofágica é uma condição relativamente incomum em pequenos animais (Glazer e Walters, 2008). De acordo com sua localização, pode ser classificada em intrínseca (intramural) ou extrínseca (extramural), abrangendo condições benignas e malignas. Estenoses intrínsecas benignas representam o estreitamento do lúmen esofágico secundário à formação de um anel de tecido fibroso cicatricial, normalmente resultado de esofagites ulcerativas

Recebido em 5 de agosto de 2016

Aceito em 10 de outubro de 2016

E-mail: marilia_luciani@hotmail.com que se aprofundam para as camadas submucosa e muscular (Adamama-Moraitou et al., 2002).

A esofagite resulta de qualquer insulto químico, físico ou mecânico sobre a mucosa esofágica. Entre suas causas estão vômito crônico, ingestão de corpos estranhos ou substâncias cáusticas, uso de determinados medicamentos - citam-se as tetraciclinas-, traumas, lesões térmicas, hérnia de hiato, agentes infecciosos - como o Pythium insidiosum - e refluxo gastroesofágico (RGE), sendo este último a principal etiologia, muitas vezes associada a procedimentos anestésicos 
(Glazer e Walters, 2008; Rodríguez-Alarcón et al., 2015; Sellon e Willard, 2003).

Uma grande variedade de tranquilizantes, sedativos e anestésicos tem a capacidade de diminuir o tônus do esfíncter esofágico inferior (EEI), parte importante do mecanismo de defesa natural do organismo e responsável por impedir o refluxo de conteúdo gástrico para o esôfago (Rodríguez-Alarcón et al., 2015).

Regurgitação é o sinal cardinal de esofagite e estenose esofágica, com associação a odinofagia, disfagia, hipersalivação, relutância em se alimentar e perda de peso progressivo. Sinais respiratórios podem estar presentes quando pneumonia aspirativa e/ou rinossinusite secundárias se desenvolvem (Glazer e Walters, 2008; Sellon e Willard, 2003). Para o diagnóstico, exames radiográficos simples e contrastados e esofagoscopia são essenciais (Adamama-Moraitou et al., 2002).

O presente relato tem por objetivo descrever as alterações clínicas, radiográficas e de esofagoscopia de duas cadelas diagnosticadas com estenose esofágica após procedimento de ovário-histerectomia (OSH), comparando seus achados aos da literatura.

Relato 1. Um canino SRD, fêmea, com um ano e pesando $5,5 \mathrm{~kg}$, foi encaminhado para atendimento no Hospital Veterinário do CAV/UDESC, com queixas de regurgitação frequente logo após a ingestão de alimento, além de emagrecimento progressivo nos últimos 20 dias, com apetite voraz, tosse e secreção nasal serosa. Os sinais iniciaram cerca de cinco dias após a realização do procedimento de OSH terapêutica, realizada havia um mês em razão de distocia e morte fetal. O protocolo anestésico era desconhecido, assim como possíveis intercorrências no perioperatório. O paciente apresentava-se caquético, severamente desidratado (índice de 10\%), hipotérmico $\left(36,8^{\circ} \mathrm{C}\right)$ e com mucosas pálidas. À ausculta havia sibilos traqueais. Foram realizadas radiografias torácica e cervical simples, nas projeções laterolateral direita e ventrodorsal, as quais demonstraram áreas de radiopacidade mista - ar e fluido - em topografia de esôfago cervical e orofaringe, sugestivas de aerofagia secundária a processo obstrutivo. Além disso, visibilizaram-se microcardia e redução da veia cava caudal, ambas compatíveis com o estado de desidratação do paciente, e pneumopatia alveolar em região cranioventral, indicativos de pneumonia aspirativa. Esofagograma foi realizado por meio da administração via oral de $15 \mathrm{~mL}$ de solução de sulfato de bário a $60 \%$, por meio do qual foi identificada uma dilatação sacular em esôfago cervical, estendendo-se a partir do esfíncter esofágico superior até a altura da quinta vértebra cervical, com adicional deslocamento ventral da traqueia, falha de preenchimento da coluna de contraste central e estreitamento do lúmen esofágico caudal à porção (Fig.1-A). Como impressão diagnóstica, concluiu-se que os principais diagnósticos diferenciais a serem considerados seriam estenose esofágica e corpo estranho esofágico. Dessa forma, foi indicada esofagoscopia, a qual demonstrou um anel de proliferação de tecido brancacento na área de estreitamento em esôfago cervical caudal, estando a mucosa adjacente hiperêmica, com úlceras e áreas de hemorragia. A partir disso, optou-se pela gastropexia com tubo para alimentação, início da antibioticoterapia e fluidoterapia, com o objetivo de melhorar a condição do paciente para posterior correção cirúrgica da estenose. Devido ao quadro crítico em que o paciente se apresentava, ele veio a óbito três dias após a gastrostomia. Na necropsia, pôde-se confirmar a ocorrência de duas áreas de estenose e esofagite, uma a $7 \mathrm{~cm}$ aboral à laringe e a outra a $5 \mathrm{~cm}$ oral do cárdia (Fig.1-B), caracterizadas por áreas de necrose da mucosa esofágica associadas a proliferação de tecido conjuntivo fibroso e infiltrado inflamatório com predominância de macrófagos. Na região de estenose mais cranial, foi possível definir um halo esbranquiçado em meio à fibrose cicatricial. 

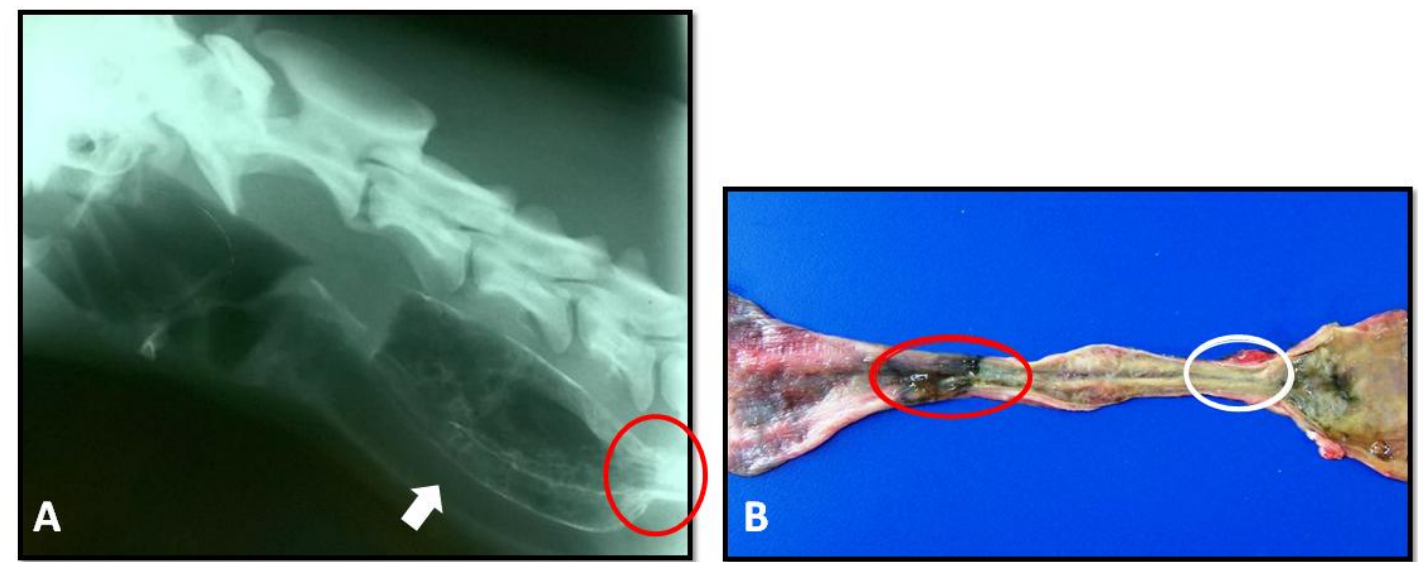

Figura 1. A) Esofagograma demonstra uma saculação em esôfago cervical (seta branca) precedendo possível área de estenose (círculo vermelho). B) Esôfago durante a necrópsia: área de $2 \mathrm{~cm}$ de estenose, distal $7 \mathrm{~cm}$ da laringe (círculo vermelho), seguida por estreitamento do lúmen e segunda área de estenose, $5 \mathrm{~cm}$ cranial à cárdia (círculo branco).

Relato 2. Um canino SRD, fêmea, com oito meses e $14,5 \mathrm{~kg}$, foi atendida no Hospital Veterinário do CAV/UDESC com queixas de regurgitação, inapetência e perda de peso. Percebeu-se ao exame físico hipersalivação, disfagia e hipertermia $\left(40,1^{\circ} \mathrm{C}\right)$. O paciente tinha passado por procedimento de OSH eletiva havia uma semana, com ocorrência de refluxo gastroesofágico no fim da anestesia e consequente pneumonia aspirativa. $\mathrm{O}$ protocolo anestésico utilizado no procedimento foi acepromazina e morfina como medicação préanestésica, propofol para indução e isoflurano como manutenção, sendo de $12 \mathrm{~h}$ o tempo médio de jejum prévio. Com suspeita de esofagite e/ou estenose esofágica, o paciente foi encaminhado para a realização de radiografia cervical simples, a qual não apontou alterações. O esofagograma foi obtido, após administração de solução de sulfato de bário a $60 \%$, por meio do qual se pôde visibilizar uma área de dilatação do lúmen esofágico cervical caudal, seguida por redução da coluna de contraste em região de esôfago torácico cranial, cranialmente à base do coração, sugerindo estenose esofágica (Fig. 2-A). Com isso, iniciou-se o tratamento clínico com utilização de omeprazol, sucralfato, metoclopramida, amoxicilina com clavulanato de potássio e prednisona, em associação ao manejo alimentar - alimentação pastosa e elevação da cabeça durante 20 minutos após a ingestão. Não obtida resposta efetiva ao tratamento imposto, seguiu-se a realização de esofagoscopia, com tentativa de dilatação esofágica por meio de uma sonda de Folley inflada. Durante o exame, foi possível visualizar a área de estreitamento esofágico em porção torácica cranial à base cardíaca, envolta por uma banda de tecido brancacento em associação a pontos discretos de mucosa hiperêmica e ulcerada (Fig. 2-B). Mesmo após o procedimento, verificou-se que a alimentação era ainda inadequada, sendo um impedimento à melhora do quadro clínico. Dessa forma, foi necessária a realização de gastrostomia para colocação de sonda de alimentação e nova dilatação esofágica. Após um mês, retirou-se a sonda de gastrostomia e seguiuse com a realização do manejo alimentar supracitado. $\mathrm{O}$ paciente apresentou melhora satisfatória, com remissão dos sinais clínicos de regurgitação e disfagia, mantendo-se o manejo alimentar ad aeternum. 

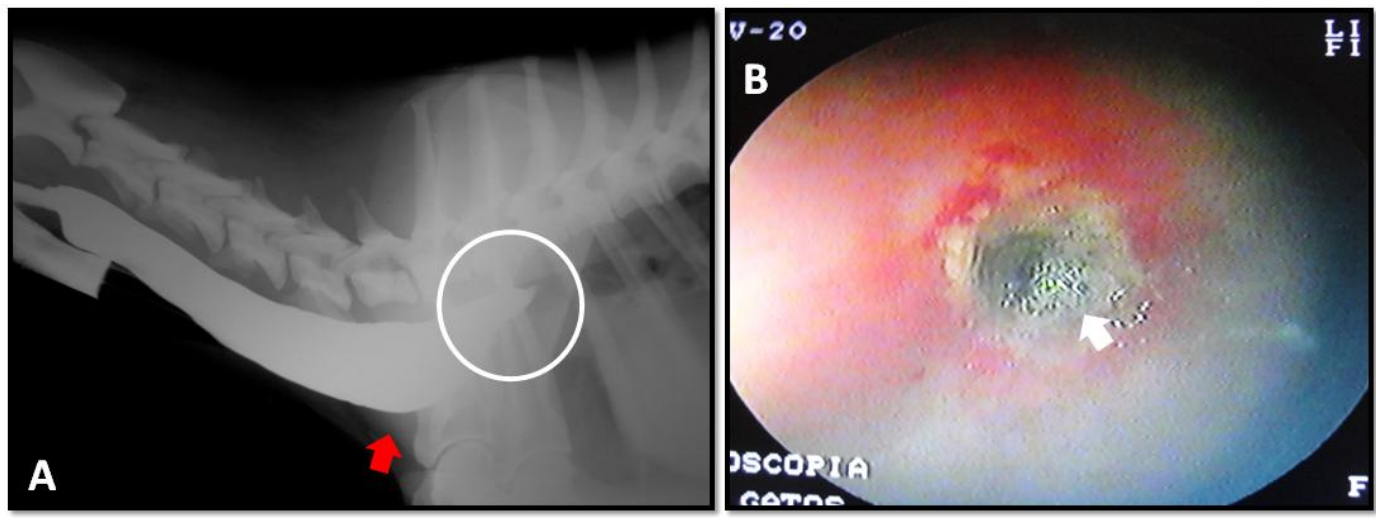

Figura 2. A) Esofagograma demonstra área de dilatação esofágica em porção cervical caudal (seta vermelha), seguida por redução do lúmen da coluna de contraste esofágico em porção intratorácica cranial (círculo). B) Esofagoscopia demonstra presença de uma banda de tecido esbranquiçado em porção de lúmen esofágico estenosado (seta branca).

\section{DISCUSSÃO}

A estenose esofágica ocorre quando um processo inflamatório ulcerativo da camada mucosa se estende para as camadas submucosa e muscular, com posterior formação de tecido conjuntivo fibroso cicatrial intraluminal que "estreita" a região, criando uma estrutura anelar de aspecto brancacento (Glazer e Walters, 2008; Sellon e Willard, 2003), semelhante àquela encontrada em necropsia no paciente do relato 1 .

O RGE transanestésico é a causa mais comum de esofagite e estenose esofágica, com incidência entre 16,3 e 65\% (Galatos e Raptopoulos, 1995'; Adamama-Moraitou et al., 2002), sendo a principal suspeita em ambos os casos. Refluxo é a passagem silenciosa do conteúdo gástrico ao longo do esôfago, facilitado durante anestesias pela redução transitória da pressão sobre o EEI (Wilson e Walshaw, 2004). Os anestésicos morfina, acepromazina, propofol e isoflurano, utilizados no segundo caso, parecem aumentar substancialmente a ocorrência de refluxo.

Wilson et al. (2005) observaram que cerca de $50 \%$ dos pacientes que receberam morfina como agente pré-anestésico apresentaram RGE. Strombeck e Harrold (1985) demonstraram grave redução da pressão do EEI após administração de acepromazina, caindo de níveis normais ao redor de $47,9 \mathrm{mmHg}$ para níveis inferiores a 18,6mmHg. Wilson et al. (2006) citaram incidência de $29 \%$ de RGE quando da realização única de acepromazina, com aumento dosedependente quando da associação com morfina.
Para os casos de RGE após indução com tiopental e propofol, demonstrou-se a ocorrência de RGE em $17,6 \%$ e $50 \%$ dos cães, respectivamente (Raptopoulos e Galatos, 1997). Os agentes inalatórios isoflurano, halotano e sevoflurano apresentam efeitos semelhantes sobre o EEI, com redução de seu tônus e aumento da incidência de RGE - 15,5\%, $21 \%$ e $20 \%$, respectivamente (Wilson et al., 2006).

RGE é descrito mais frequentemente após procedimentos cirúrgicos intra-abdominais (Galatos e Raptopoulos, $1995^{1}$ ), com incidência de até $47 \%$ em cães (Leib et al., 2001), enquanto nos demais procedimentos a ocorrência de lesão esofágica gira em torno de $17 \%$ (Wilson e Walshaw, 2004), incluindo procedimentos ortopédicos, odontológicos e cirurgias de tecidos moles extra-abdominais. $\mathrm{O}$ aumento da pressão intra-abdominal, com consequente redução relativa da pressão do EEI, é descrito como um dos fatores predisponentes (Rodríguez-Alarcón et al., 2015), sendo o fator possivelmente envolvido na lesão esofágica ocorrente após os procedimentos relatados.

Adamama-Moraitou et al. (2002) diagnosticaram a estenose esofágica após procedimentos intraabdominais em 14 dos 20 casos relatados, em que 12 eram de cirurgias do aparelho reprodutor. A alta incidência de estenose esofágica após cirurgias obstétricas pode ser explicada pelo fato de a OSH ser um dos procedimentos mais comuns na rotina veterinária. Contudo, altos níveis de progesterona presentes na gestação causam significativa redução da pressão do EEI, 
além de que a alta pressão exercida pelo útero de volume aumentado sobre o estômago soma-se ao tônus reduzido (Adamama-Moraitou et al., 2002). O primeiro caso descrito está relacionado à distocia, podendo tais fatores terem contribuído para a ocorrência da lesão esofágica posteriormente à $\mathrm{OSH}$.

De acordo com diversos estudos e conforme demonstrado no presente trabalho, fêmeas parecem ter maior risco de desenvolvimento de estenose esofágica pós-anestésicas. Além da progesterona, o estrógeno parece também agir sobre o EEI, reduzindo seu tônus e predispondo ao RGE (Adamama-Moraitou et al., 2002; Rodríguez-Alarcón et al., 2015; Wilson e Walshaw, 2004).

Diversos fatores são determinantes para o desenvolvimento de esofagite e de estenose após o episódio de RGE, assim como para a gravidade e a localização das lesões (Wilson e Walshaw, 2004). Além de falhas no sistema antirrefluxo, em especial sobre o tônus do EEI, a capacidade de "autolimpeza" e a resistência da mucosa esofágica sobre o conteúdo gástrico são decisivas (Adamama-Moraitou et al., 2002).

O tempo de contato e a composição do conteúdo expelido, sobretudo sua acidez, presença de sais biliares e enzimas (como pepsina e tripsina), são fatores especialmente danosos à mucosa (Rodríguez-Alarcón et al., 2015). Wilson e Walshaw (2004) descreveram lesões da mucosa esofágica após o contato com conteúdo de $\mathrm{pH}$ menor que 2,5 por período menor que 20 minutos. Galatos e Raptopoulos (1995a) demonstraram que $10 \%$ dos 270 casos de RGE avaliados eram de $\mathrm{pH}$ ácido menor que 2,5, sendo $98,5 \%$ das ocorrências de $\mathrm{pH}$ ácido menor que 4,5 .

Discretas lesões esofágicas causadas pelo conteúdo gástrico regurgitado levam a um ciclo vicioso de danos: inicialmente, há redução da motilidade do órgão com consequente falhas na eliminação de substâncias irritantes; assim, desenvolve-se processo inflamatório, que por fim diminui a pressão do EEI e predispõe à ocorrência de RGE, culminando na intensificação e expansão gradativa das lesões à mucosa e na progressão da inflamação local (Glazer e Walters, 2008; Sellon e Willard, 2003). Jejum prolongado, por mais de $12 \mathrm{~h}$, aumenta a acidez gástrica e, consequentemente, a ocorrência de lesões de esôfago pós-anestésicas (Galatos e Raptopoulos, 1995b), possivelmente o ocorrido no relato dois.

A estenose esofágica pós-refluxo pode ocorrer em qualquer porção de seu trajeto, dependendo do volume expelido e da extensão de alcance (Leib et al., 2001). Estenoses em esôfago cervical, como aquela do relato 1 , são menos frequentes, com incidência de até $22 \%$ (Leib et $a l .$, 2001). Já aquelas em esôfago torácico cranial à base do coração, semelhante ao caso 2, são citadas em $32 \%$ dos casos (Leib et al., 2001). O esôfago torácico caudal à base do coração é a região mais comumente acometida e, quando associado à estenose do esôfago cervical como presente no primeiro relato, pode ter incidência de até $75 \%$ (Adamama-Moraitou et al., 2002). Apesar de autores citarem a interferência do decúbito do paciente durante a cirurgia sobre a ocorrência de RGE (Adamama-Moraitou et al., 2002), Galatos e Raptopoulos (1995a) não encontraram diferenças significativas quanto a esses fatores em 270 casos avaliados.

Os sinais clínicos de estenose esofágica desenvolvem-se, em média, uma a duas semanas após a lesão inicial (Leib et al., 2001), variando entre zero e 21 dias (Wilson e Walshaw, 2004). Nos pacientes relatados, os sinais clínicos iniciaram dentro de cinco e sete dias após a OSH, sendo a regurgitação a principal queixa relacionada. Regurgitação é o sinal cardinal de esofagite e estenose esofágica (Sellon e Willard, 2003), presente em $100 \%$ dos 28 casos descritos por Wilson e Walshaw (2004), com gravidade proporcional à extensão e profundidade dos danos à parede esofágica. Emagrecimento progressivo com manutenção de apetite são comuns em casos graves (Glazer e Walters, 2008), associado à hipersalivação, disfagia e odinofagia (clinicamente avaliada como dor ao engolir, com tentativas repetidas de deglutição ou até mesmo relutância em deglutir) (Adamama-Moraitou et al., 2002; Wilson e Walshaw, 2004), como visto nos casos aqui descritos. Sinais respiratórios, como tosse e secreção nasal, estão relacionados à pneumonia por aspiração ou rinossinusite secundária à regurgitação (Glazer e Walters, 2008), presentes no primeiro relato. Febre, existente no segundo paciente, ocorre pelo processo inflamatório 
esofágico ou por doença respiratória secundária (Sellon e Willard, 2003).

O diagnóstico definitivo requer a demonstração das anormalidades esofágicas pelo esofagograma e pela esofagoscopia (Sellon e Willard, 2003). Além dos sinais clínicos e de histórico sugestivos de doença esofágica, pacientes com pneumonia recorrente devem ser avaliados quanto à presença de afecções de esôfago (Glazer e Walters, 2008).

O exame radiográfico anterior à endoscopia é importante para exclusão de outras causas de regurgitação, como corpos estranhos ou neoplasias (Sellon e Willard, 2003); para detecção de alterações sugestivas de pneumonia aspirativa, presentes no primeiro relato, como pneumopatia alveolar cranioventral; e para identificação do número, da localização e da extensão dos sítios de estenose existentes, já que a esofagoscopia não permite a avaliação após o primeiro sítio de estreitamento (Glazer e Walters, 2008). Radiografias simples são pouco sensíveis na detecção de doença esofágica, contudo a aerofagia é um achado comum (Wilson e Walshaw, 2004).

$\mathrm{Na}$ esofagoscopia, áreas de estenose são detectadas quando há redução do lúmen esofágico em cerca de $50 \%$ em relação ao diâmetro normal, com mucosa revestida por tecido fibroso esbranquiçado, liso e brilhante (Leib et al., 2001), semelhante às regiões visibilizadas nos pacientes relatados. $\mathrm{O}$ exame endoscópico é a modalidade mais sensível para diagnóstico de esofagite, demonstrando áreas de lúmen preservado com mucosa de aspecto granular, hiperêmico e contendo erosões, úlceras ou pontos de hemorragia (Leib et al., 2001; Sellon e Willard, 2003), como a imagem encontrada no exame de esofagoscopia do primeiro paciente relatado.

O tratamento inicial consiste em proteger a mucosa esofágica de danos adicionais (Sellon e Willard, 2003) e manter o suporte nutricional do paciente. O uso de tubos de alimentação via gastrostomia, como utilizado em ambos os casos, parece ser satisfatório por esses auxiliarem na redução de insultos à parede esofágica, além de permitirem melhor manejo alimentar (Glazer e Walters, 2008). Antagonistas de receptores H2 e inibidores da bomba de prótons são recomendados a fim de reduzir a acidez gástrica, em associação a protetores de mucosa e antieméticos (Glazer e Walters, 2008; Sellon e Willard, 2003), conforme realizado no paciente do relato 2, no qual omeprazol, sucralfato e metoclopramida foram utilizados. Glicocorticoides parecem auxiliar de maneira eficiente no controle do processo inflamatório local (Glazer e Walters, 2008; Sellon e Willard, 2003).

Estenoses esofágicas normalmente requerem dilatação para regressão dos sinais clínicos (Glazer e Walters, 2008). O procedimento pode ser cirúrgico, basicamente por ressecção e anastomose da região de estreitamento, assim como por meio de dilatação via cateteres de balão, velas ou tubos endotraqueais guiados pela esofagoscopia (Adamama-Moraitou et al., 2002). No relato 2, técnica semelhante à dilatação por cateter de balão foi realizada, adaptando-se para isso uma sonda de Folley.

O prognóstico em geral é bom, com resolução dos sinais clínicos em 79 a 88\% dos casos, segundo Glazer e Walters (2008), conforme acompanhado no paciente do relato 2 . O paciente do relato 1 veio a óbito antes mesmo da intervenção direta sobre a área de estenose, possivelmente devido ao grave estado de desnutrição e desidratação em que se encontrava no momento do atendimento veterinário.

\section{AGRADECIMENTOS}

À CAPES pelo apoio financeiro disponibilizado para realização da presente publicação.

\section{CONCLUSÃO}

Os sinais de regurgitação, disfagia e emagrecimento progressivo após a realização do procedimento de $\mathrm{OSH}$, em ambo os casos, foi sugestivo da ocorrência de esofagite e estenose esofágica, ainda que o fenômeno RGE não tenha sido registrado no transanestésico do primeiro relato. $\mathrm{O}$ esofagograma e a esofagoscopia foram essenciais para a definição do diagnóstico, demonstrando os sítios de estenose $\mathrm{e}$ identificando áreas de inflamação da mucosa esofágica. A dilatação esofágica guiada por esofagoscopia, associada ao suporte nutricional e à terapia medicamentosa antiácida, demonstrou ser eficaz para o tratamento da afecção no segundo paciente. 


\section{REFERÊNCIAS}

ADAMAMA-MORAITOU, K.K.; RALLIS, T.S.; PRASSINOS, N.N. et al. Benign esophageal stricture in the dog and cat: a retrospective study of 20 cases. Can. J. Vet. Res., v.66, p.55-59, 2002.

GALATOS, A.D.; RAPTOPOULOS, D. Gastrooesophageal reflux during anaesthesia in the dog: the effect of age, positioning and type of surgical procedure. Vet. Rec., v.20, p.513-516, 1995a.

GALATOS, A.D.; RAPTOPOULOS, D. Gastrooesophageal reflux during anaesthesia in the dog: the effect of preoperative fasting and premedication. Vet. Rec., v.19, p.479-483, $1995 b$.

GLAZER, A.; WALTERS, P. Esophagitis and esophageal strictures. Comp. Cont. Educ. Vet., v.30, p.281-292, 2008.

LEIB, M.S. et al. Endoscopic balloon dilation of benign esophageal strictures in dogs and cats. $J$. Vet. Intern. Med., v.15, p.547-552, 2001.

RAPTOPOULOS, D.; GALATOS, A.D. Gastrooesophageal reflux during anaesthesia induced with either thiopentone or propofol in the dog. $J$. Vet. Anaesth., v.24, p.20-23, 1997.
RODRÍGUEZ-ALARCÓN, C.A.; BERISTAINRUIZ, D.M.; RIVERA-BARRENO, R. et al. Gastroesophageal reflux in anesthetized dogs: a review. Rev. Colomb. Cienc. Pecu., v.28, p.144155,2015

SELLON, R.K.; WILLARD, M.D. Esophagitis and esophageal strictures. Vet. Clin. Small Anim., v.33, p.945-967, 2003.

STROMBECK, D.R.; HARROLD, D. Effects of atropine, acepromazine, meperidine, and xylanize on gastroesophageal sphincter pressure in the dog. Am. J. Vet. Res., v.46, 963-965, 1985.

WILSON, D.V.; BORUTA, D.T.; EVANS, A.T. Influence of halothane, isoflurane, and sevoflurane on gastroesophageal reflux during anesthesia in dogs. Am. J. Vet. Res., v.67, p.1821-1825, 2006.

WILSON, D.V.; EVANS, A.T.; MILLER, R. Effects of preanesthetic administration of morphine on gastroesophageal reflux and regurgitation during anesthesia in dog. Am. J. Vet. Res., v.66, p.386-390, 2005.

WILSON, D.V.; WALSHAW, R. Postanesthetic esophageal dysfunction in 13 dogs. J. Am. Anim. Hosp. Assoc., v.40, p.4. 